\title{
Análise de séries temporais de focos de calor em biomas brasileiros utilizando o método Gráfico de Recorrência
}

\author{
Analysis of time series of hot pixels in brazilian biomes using the Recurrence Plot method \\ Análisis de series temporales de focos de calor en biomas brasileños utilizando el método Gráfico de
} Recurrencia

Recebido: 15/03/2021 | Revisado: 21/03/2021 | Aceito: 26/03/2021 | Publicado: 04/04/2021

Vaniele da Silva Barros

ORCID: https://orcid.org/0000-0002-3238-1106 Universidade Federal Rural de Pernambuco, Brasil E-mail: vaniiellebarros@hotmail.com

Leika Irabele Tenório de Santana

ORCID: https://orcid.org/0000-0002-5516-5887 Universidade Federal Rural de Pernambuco, Brasil E-mail: leikatenorio10@gmail.com

Joelma Mayara da Silva

ORCID: https://orcid.org/0000-0003-2354-6068 Universidade Federal Rural de Pernambuco, Brasil E-mail: jms.estat@gmail.com

Lidiane da Silva Araújo

ORCID: https://orcid.org/0000-0003-3111-7925 Universidade Federal Rural de Pernambuco, Brasil E-mail: lidiane_sac@hotmail.com

Cristiane Rocha Albuquerque ORCID: https://orcid.org/0000-0002-4955-8131 Universidade Federal Rural de Pernambuco, Brasil E-mail: ita.chris@gmail.com

Tatijana Stosic

ORCID: https://orcid.org/0000-0002-5691-945X Universidade Federal Rural de Pernambuco, Brasil E-mail: tastosic@gmail.com

\begin{abstract}
Resumo
As queimadas são processos complexos que podem ter causas naturais ou antrópicas, e seus efeitos num ecossistema variam de acordo com sua sensibilidade. A recorrência de focos de incêndio pode afetar o equilíbrio ambiental e a saúde humana. Assim, faz-se necessário monitorar a ocorrência do fogo e compreender sua dinâmica. No Brasil, o monitoramento é feito via satélite, com que se detectam os focos de calor, trabalho este realizado pelo Instituto Nacional de Pesquisas Espaciais (INPE). Com o objetivo de investigar a variabilidade temporal das queimadas nos biomas Amazônia, Cerrado, Caatinga e Mata Atlântica, utilizou-se o método Gráfico de Recorrência e a Análise de Quantificação de Recorrência, desenvolvidos para o estudo da dinâmica não linear de séries temporais. Foram então analisadas as séries diárias de focos de calor nesses biomas geradas a partir de dados disponibilizados pelo INPE, registrados entre julho/2002 e dezembro/2019. Os gráficos de recorrência de focos de calor para os biomas Amazônia e Cerrado mostraram que seus sistemas dinâmicos de queimadas possuem parâmetros que variam lentamente e são não-estacionários. Observou-se também estrutura semiperiódica para o bioma Caatinga, e descontínua para o bioma Mata Atlântica, indicando, para este último, mudanças repentinas na dinâmica. Além disso, de acordo com a Análise de Quantificação de Recorrência, a série temporal de queimadas no bioma Mata Atlântica apresentou a menor previsibilidade e o maior grau de desordem, enquanto o bioma Caatinga revelou série temporal de focos de calor mais previsível e com dinâmica mais estável.
\end{abstract}

Palavras-chave: Queimadas; Biomas; Gráfico de recorrência; Análise de quantificação de recorrência.

\begin{abstract}
Vegetation fires are complex processes that can have natural or man-made causes, and their effects on an ecosystem vary according to its sensitivity. The recurrence of fire outbreaks can affect the environmental balance and human health. Thus, it is necessary to monitor the occurrence of fire and understand its dynamics. In Brazil, monitoring is carried out via satellite, with which hot pixels are detected. This process is accomplished by the National Institute for Space Research (INPE). In order to investigate the temporal variability of fires in the Amazon, Cerrado, Caatinga and Atlantic Forest biomes, the Recurrence Plot method and the Recurrence Quantification Analysis, developed to study the nonlinear dynamics of time series, were used. So, the daily series of hot pixels in these biomes, generated from
\end{abstract}


data made available by INPE, recorded between July/2002 and December/2019, were analyzed. The recurrence plots of hot pixels for the Amazon and Cerrado biomes showed that their dynamic systems of vegetation fires have parameters that vary slowly and are non-stationary. It was also observed a semi-periodic structure for the Caatinga biome and discontinuous for the Atlantic Forest biome, indicating, in the latter, sudden changes in the dynamics. Besides that, according to the Recurrence Quantification Analysis, the time series of vegetation fires in the Atlantic Forest biome presented the least predictability and the highest degree of disorder, while the Caatinga biome revealed a more predictable hot pixels time series with more stable dynamics.

Keywords: Vegetation fires; Biomes; Recurrence plot; Recurrence quantification analysis.

\section{Resumen}

Los incendios forestales son procesos complejos que pueden tener causas naturales o provocadas por el hombre, y sus efectos sobre un ecosistema varían según su sensibilidad. La recurrencia de incendios puede afectar el equilibrio ambiental y la salud humana. Así, es necesario monitorear la ocurrencia de incendios y comprender su dinámica. En Brasil, el seguimiento se realiza vía satélite, con que se detectan focos de calor, y este proceso lo lleva a cabo el Instituto Nacional de Pesquisas Espaciales (INPE). Para investigar la variabilidad temporal de incendios en los biomas Amazonia, Cerrado, Caatinga y Mata Atlántica, se utilizó el método Gráfico de Recurrencia y el Análisis de Cuantificación de Recurrencia, desarrollados para estudiar la dinámica no lineal de series temporales. Luego, se analizaron las series diarias de focos de calor en estos biomas generadas a partir de datos puestos a disposición por el INPE, registrados entre julio/2002 y diciembre/2019. Los gráficos de recurrencia de focos de calor en los biomas Amazonia y Cerrado mostraron que sus sistemas dinámicos de incendio tienen parámetros que varían lentamente y son no estacionarios. También se observó estructura semi-periódica para el bioma de Caatinga y discontinua para el bioma de Mata Atlántica, indicando, en este último, cambios repentinos en la dinámica. Además, según el Análisis de Cuantificación de Recurrencia, la serie temporal de incendios en el bioma Mata Atlántica mostró la menor previsibilidad y el mayor grado de desorden, y el bioma Caatinga reveló serie temporal de focos de calor más predecible con dinámica más estable.

Palabras clave: Incendios forestales; Biomas; Gráfico de recurrencia; Análisis de cuantificación de recurrencia.

\section{Introdução}

O fogo é um fenômeno mundial que influencia os padrões e processos globais dos ecossistemas, incluindo a distribuição e estrutura da vegetação, o ciclo do carbono e o clima. De acordo com a fundação NSF - National Science Foundation (2003), dois dos maiores desafios das Ciências Ambientais nas primeiras décadas do século XXI são: proteção do meio ambiente e diminuição das consequências dos desastres físico-naturais, que têm se intensificado com as atividades humanas.

No que se refere às queimadas e, principalmente, aos incêndios florestais, além de liberarem na atmosfera grande quantidade de gases responsáveis pelo efeito estufa, são uma das maiores ameaças à preservação da biodiversidade do planeta, com impactos diretos sobre a fauna e a flora, e ainda contribuem, indiretamente, com a degradação ambiental (Soares \& Batista, 2007). Além disso, a queima da biomassa vegetal pode gerar problemas para a saúde humana, especialmente doenças respiratórias (Arbex, Cançado, Pereira, Braga, \& Saldiva, 2004), e pode provocar consequências socioeconômicas consideráveis.

Atualmente, a existência de milhares de focos de incêndios no Brasil tem sido frequentemente noticiada pela imprensa, principalmente durante a estação mais seca, geralmente de julho a outubro (Gontijo, Pereira, Oliveira, \& Júnior, 2011). Entre 2002 e 2019 o Instituto Nacional de Pesquisas Espaciais (INPE) registrou mais de 4 milhões de dados associados à ocorrência de temperaturas acima de $47^{\circ} \mathrm{C}$, capturados pelos satélites de monitoramento e identificados como focos de calor. A detecção sistemática de focos de calor realizada pelo INPE é pioneira e a mais completa desenvolvida no mundo. De acordo com a coordenação do Programa de Queimadas do Instituto Nacional, as tecnologias para o monitoramento de queimadas por meio de imagens de satélites vêm sendo aprimoradas desde 1980. Isso é particularmente útil em regiões remotas sem meios intensivos de acompanhamento, condição que representa a situação geral do Brasil.

Aspectos naturais in loco, bem como algumas variáveis antrópicas podem contribuir significativamente para a incidência e propagação do fogo (Archibald, Roy, Wilgen, \& Scholes, 2009). Fatores como vegetação, condições climáticas e 
medidas de proteção, entre outros, podem afetar a iniciação, propagação e supressão de incêndios, tornando extremamente difícil a modelagem desse fenômeno. Por isso, há um esforço contínuo da comunidade científica em desenvolver modelos teóricos e computacionais mais eficazes para descrever a disseminação do fogo e, assim, possibilitar a criação de procedimentos mais eficientes de manejo de recursos bioambientais, uso do solo e agricultura (Bowman, Balch, Artaxo, Bond, Carlson, Cochrane, \& Pyne, 2009).

Nesse sentido, faz-se necessário compreender melhor o sistema complexo das queimadas nos diferentes biomas brasileiros, especialmente o nível de previsibilidade da variação temporal. Ele será tratado neste trabalho pelo método Gráfico de Recorrência (Recurrence Plot - RP) e pela Análise de Quantificação de Recorrência (Recurrence Quantification Analysis RQA), que foram desenvolvidos para a análise não linear das séries temporais, baseando-se nas recorrências dos estados de sistemas dinâmicos no espaço de fase (Marwan, Romano, Thiel, \& Kurths, 2007; Zbilut; Webber JR, 1992).

Nas últimas décadas, os gráficos de recorrência tornaram-se uma alternativa para o estudo de sistemas complexos, e isso pode ser constatado por sua aplicação em áreas como Engenharia (Faustine, Pereira, \& Klemenjak, 2020), Medicina (Afonso, Rosa, Pereira, Weber, Hook, Albuquerque, \& Papa, 2019), Geofísica (Marwan, Thiel, \& Nowaczyk, 2002; Donner, Balasis, Stolbova, Georgiou, Wiedermann, \& Kurths, 2019), Química (Rustici, Caravati, Petretto, \& Marchettini 1999), Climatologia (Goswami, Marwan, Feulner, \& Kurths, 2013) e Economia (Addo, Billio, \& Guegan, 2013; Bastos \& Caiado, 2011; de Santana, da Silva, Araújo, Moreira, \& Stosic, 2020).

\section{Metodologia}

O procedimento aqui realizado para estudar o comportamento dos sistemas dinâmicos que governam as séries temporais de interesse tem como base a análise qualitativa dos dados, a partir do método Gráfico de Recorrência (Eckmann, Kamphorst, \& Ruelle, 1987), e a análise quantitativa, por meio da Análise de Quantificação de Recorrência (Zbilut \& Webber Jr., 1992), com que são obtidas as medidas de quantificação de recorrência. Isto é, as séries temporais são transformadas em redes complexas e, a partir delas, geram-se gráficos de recorrência. Deles, extraem-se as medidas de quantificação de recorrência, a partir das quais se pode distinguir entre diferentes tipos de dinâmicas não lineares e, assim, classificar o comportamento dinâmico do sistema envolvido. Essas técnicas, descritas mais adiante nesta seção, foram aqui aplicadas com uso do software R Core Team (2020).

\subsection{Descrição dos dados}

Neste trabalho são analisados os dados de focos de calor nos biomas brasileiros Amazônia, Cerrado, Mata Attântica e Caatinga, detectados pelo satélite de referência AQUA_M-T entre 04 de julho de 2002 e 31 de dezembro de 2019. Os dados foram coletados e disponibilizados pelo INPE. As séries temporais de focos de calor geradas a partir deles são apresentadas na Figura 1. 
Figura 1. Séries temporais anuais do número de focos de calor nos biomas brasileiros Amazônia, Caatinga, Cerrado e Mata Atlântica.

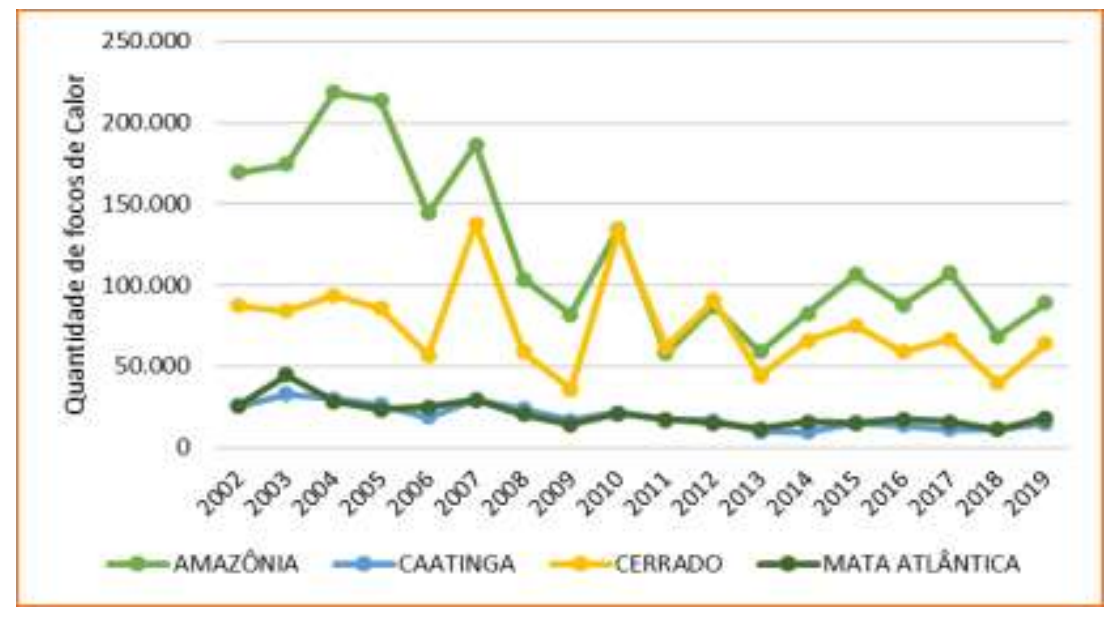

Fonte: Autores, a partir de dados do INPE (2021).

\subsection{O método Gráfico de Recorrência}

O Gráfico de Recorrência (Recurrence Plot - RP) foi introduzido por Eckmann, Kamphorst e Ruelle (1987) como uma forma de visualizar a recorrência de sistemas dinâmicos unidimensionais ou multidimensionais num espaço abstrato, denominado espaço de fase. O método viabiliza a análise bidimensional do comportamento desses sistemas, que são representados por séries temporais.

O espaço de fase é um espaço matemático formado por coordenadas que representam todas as variáveis necessárias para especificar o estado instantâneo do sistema e é uma ferramenta útil na compreensão do comportamento de sistemas dinâmicos. O estado do sistema num dado instante é representado por um ponto, e a sua evolução temporal, por uma trajetória no espaço de fase (Marwan et al., 2007). A partir do Gráfico de Recorrência, é possível analisar a trajetória do espaço da fase $m$-dimensional por meio da representação bidimensional.

O RP pode ser expresso matematicamente pela matriz quadrada $N \times N$ dada por

$$
R_{i j}(\varepsilon)=\Theta\left(\varepsilon-\left\|\vec{x}_{i}-\vec{x}_{j}\right\|\right), \operatorname{com} \vec{x}_{i} \in \mathfrak{R}^{m} \text { e } i, j=1, \ldots, N
$$

em que $\vec{x}_{i}$ e $\vec{x}_{j}$ são vetores no espaço de fase e representam a evolução dos estados do sistema dinâmico, $\varepsilon$ é o limiar (threshold), um parâmetro que determina se os estados são considerados recorrentes ou não recorrentes, $\Theta(\cdot)$ é a função de Heaviside, que mapeia os pontos recorrentes dentro do gráfico de recorrência, $\|\cdot\|$ é a norma (mínima, máxima ou euclidiana) e $m$ é a dimensão de imersão. Para uma série temporal escalar $\left\{u_{i}\right\}$, os vetores do espaço de fase são construídos por $\vec{x}_{i}=\left(u_{i}, u_{i+\tau}, \ldots, u_{i+(m-1) \tau}\right)$, em que $m$ é a dimensão de imersão e $\tau$ é o tempo de retardo. Se $R_{i j}=1$, traça-se um ponto preto nas coordenadas $(i, j)$ e, se $R_{i j}=0$, traça-se um ponto branco. Os parâmetros dimensão de imersão $m$ e tempo de retardo $\tau$ podem ser obtidos utilizando o método dos falsos vizinhos mais próximos e a função de informação mútua, respectivamente (Cao, 1997).

Um gráfico de recorrência pode apresentar diferentes padrões visuais a partir dos quais é possível extrair informações sobre a série analisada. Segundo Eckmann, Kamphorst e Ruelle (1987), esses padrões podem ser divididos em duas categorias: larga escala - tipologia (typology), e pequena escala - textura (texture). Os padrões de larga escala fornecem uma visão global 
do comportamento do sistema (ruidoso, periódico etc.), enquanto os padrões de pequena escala fornecem as estruturas do gráfico (Marwan \& Kurths, 2005).

A tipologia pode ser caracterizada como Homogênea, Periódica, Deriva e Descontinua (Eckmann, Kamphorst, \& Ruelle, 1987; Marwan, 2003; Marwan et al., 2007), conforme apresentado na Figura 2 e descrito a seguir.

- Homogênea: os pontos são pequenos se forem comparados ao gráfico em sua totalidade, e isso dificulta a identificação de quaisquer padrões na disposição das observações (Figura 2a).

- Periódica: tem como característica a presença de linhas diagonais paralelas à LOI (linha diagonal principal com $R_{i i}=1$ ), totalmente preenchidas, possuem estruturas de blocos recorrentes com aspecto de tabuleiro de xadrez e são características de sistemas oscilantes (Figura 2b).

- Deriva: é característica de sistemas em que os parâmetros variam lentamente. Apresenta ausência de pontos recorrentes tanto no canto superior esquerdo quanto no inferior direito; ou seja, os pontos recorrentes diminuem à medida que se afastam da LOI (Figura 2c).

- Descontínua: o RP com essa tipologia é característico de sistemas em que a descontinuidade é causada por mudanças abruptas na dinâmica ou pela ocorrência de eventos raros. Apresenta faixas brancas provocadas por essas mudanças ou por eventos extremos (Figura 2d).

Figura 2. Exemplo das tipologias do RP: (a) Homogênea, (b) Periódica (c) Deriva e (d) Descontinua.

(a)

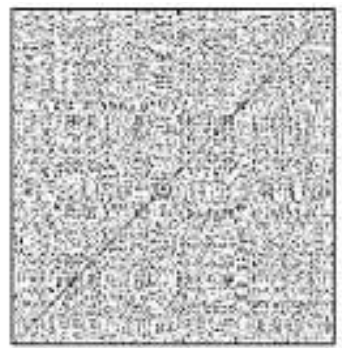

(b)

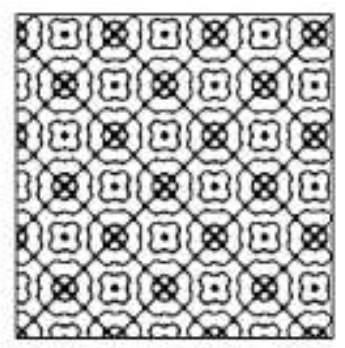

(c)

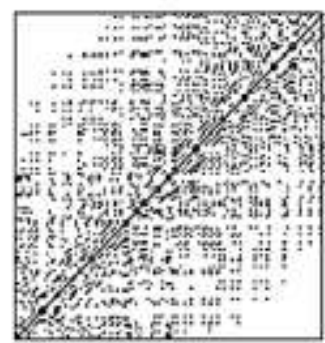

(d)

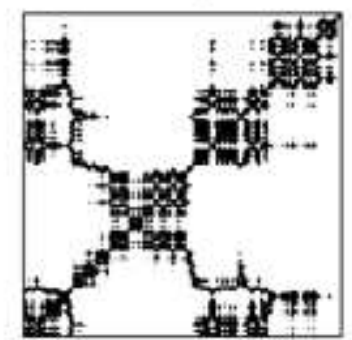

Fonte: Adaptada de (Marwan et al., 2007).

Inspeção mais detalhada do Gráfico de Recorrência está presente na estrutura da categoria textura (pequena escala), que é caracterizada por pontos únicos e isolados, linhas diagonais, e linhas verticais e horizontais (Marwan, 2003), a saber:

- Pontos únicos $\boldsymbol{e}$ isolados: representam um estado recorrente $\left(R_{i j}=1\right)$ e podem ser interpretados como estados que não persistem por um longo período.

- Linhas diagonais: ocorrem quando uma parte da trajetória evolui de forma paralela a outro segmento de trajetória, indicando que a trajetória visita a mesma região do espaço de fase em tempos diferentes.

- Linhas verticais e horizontais: ocorrem quando o estado do sistema permanece estacionário durante sua evolução temporal (Marwan, 2003; Facchini, Mocenni, Marwan, \& Tiezzi, 2007).

\subsection{Análise de Quantificação de Recorrência}

Zbilut e Webber Jr. (1992) desenvolveram a Análise de Quantificação de Recorrência (Recurrence Quantification Analysis - RQA) (Zbilut \& Webber Jr, 1992; Zbilut, Giuliani, \& Webber Jr, 1998), com que são obtidas as medidas de quantificação de recorrência (também conhecidas como medidas de complexidade), que permitem a quantificação da estrutura dos RPs. Essas medidas são obtidas com base na densidade de pontos de recorrência e nas estruturas de linhas diagonais e 
verticais do gráfico (Marwan et al., 2007). As expressões que as definem e que são descritas a seguir foram todas extraídas de (Zbilut \& Webber Jr., 1992).

A primeira medida, que quantifica a densidade de pontos de recorrência no RP, é denominada taxa de recorrência (Recurrence Rate $-R R$ ) e corresponde à probabilidade de ocorrência de um estado no espaço de fase, dentro de um limiar escolhido. A taxa de recorrência $R R$ pode variar entre $0 \%$ (não há pontos recorrentes) e $100 \%$ (todos os pontos são recorrentes), e é representada pela seguinte expressão:

$$
R R(\varepsilon)=\frac{1}{N^{2}} \sum_{i, j=1}^{N} R_{i j}(\varepsilon) .
$$

A segunda, é denominada determinismo (Determinism - DET). Essa medida, também conhecida como previsibilidade, é obtida pela razão entre o número de pontos pertencentes às diagonais e o número total de pontos recorrentes. Isto é:

$$
D E T=\frac{\sum_{l=l_{\min }}^{N} l P(l)}{\sum_{l=1}^{N} l P(l)},
$$

em que $P(l)$ representa a probabilidade de existência de linhas diagonais de comprimento $l$, e $l_{\min }$ é o menor tamanho que uma linha deve ter para ser considerada diagonal. Para um sistema de comportamento não correlacionado ou fracamente correlacionado, estocástico ou caótico, as linhas diagonais serão nulas ou muito curtas. Para sistemas periódicos, as linhas diagonais serão mais longas e com menos pontos de recorrência isolados.

Outra medida de quantificação é o tamanho médio das linhas diagonais (Average diagonal line length - L), que fornece o tempo médio em que dois segmentos da trajetória permanecem evoluindo de maneira similar em um estado do sistema. Pode ser definido como o tempo médio de previsibilidade do sistema e é expresso por

$$
L=\frac{\sum_{l=l_{\min }}^{N} l P(l)}{\sum_{l=l_{\min }}^{N} P(l)} .
$$

Calcula-se também o comprimento máximo da linha diagonal (Longest diagonal line - $L_{\max }$ ), que se refere ao comprimento máximo da linha diagonal no RP, excluindo a linha diagonal principal de identidade, LOI $(i=j)$. Matematicamente, é dada por:

$$
L_{\max }=\max \left(\left\{l_{i}\right\}_{i=1}^{N_{l}}\right),
$$

onde $N_{l}$ é o número total de linhas diagonais. O inverso dessa medida está relacionado à divergência exponencial (Divergence - DIV) da trajetória do espaço de fase. Isto é:

$$
D I V=\frac{1}{L_{\max }} .
$$

Assim, quanto mais rápido os segmentos de trajetória divergem, mais curtas são as linhas diagonais e mais altos são os valores da medida $D I V$.

As medidas de RQA baseadas nas estruturas verticais e horizontais dos RPs são: laminaridade (Laminarity - LAM) e tempo de permanência (Trapping Time - TT). A laminaridade é a razão entre os pontos de recorrência que formam a estrutura e todo o conjunto de pontos de recorrência que podem ser calculados. Ela é definida por 


$$
L A M=\frac{\sum_{v=v_{\min }}^{N} v P(v)}{\sum_{v=1}^{N} v P(v)},
$$

em que $P(v)$ representa a probabilidade de existência de linhas verticais de tamanho $v$ e $v_{\min }$ corresponde ao tamanho mínimo da linha vertical a ser considerada. O tempo de permanência $T T$, por sua vez, é obtido calculando-se o comprimento médio das estruturas verticais. Ele estima o tempo médio em que o sistema permanecerá num determinado estado e é dado por

$$
T T=\frac{\sum_{v=v_{\min }}^{N} v P(v)}{\sum_{v=v_{\min }}^{N} P(v)},
$$

com $P(v), v$ e $v_{\min }$ já definidos na expressão (7) para a medida $L A M$.

\section{Resultados e Discussão}

Para gerar as redes complexas a serem analisadas, as séries temporais originais de focos de calor de cada bioma foram normalizadas entre 0 e 1 , de acordo com a expressão

$$
Y=\frac{x-\min (x)}{\max (x)-\min (x)},
$$

em que $x$ denota a série temporal original, e $\max (x)$ e $\min (x)$ são os valores de máximo e mínimo de $x$, respectivamente (Bastos \& Caiado, 2011). As séries temporais normalizadas de focos de calor para cada bioma são apresentadas na Figura 3.

Figura 3. Séries temporais normalizadas do número de focos de calor por bioma.
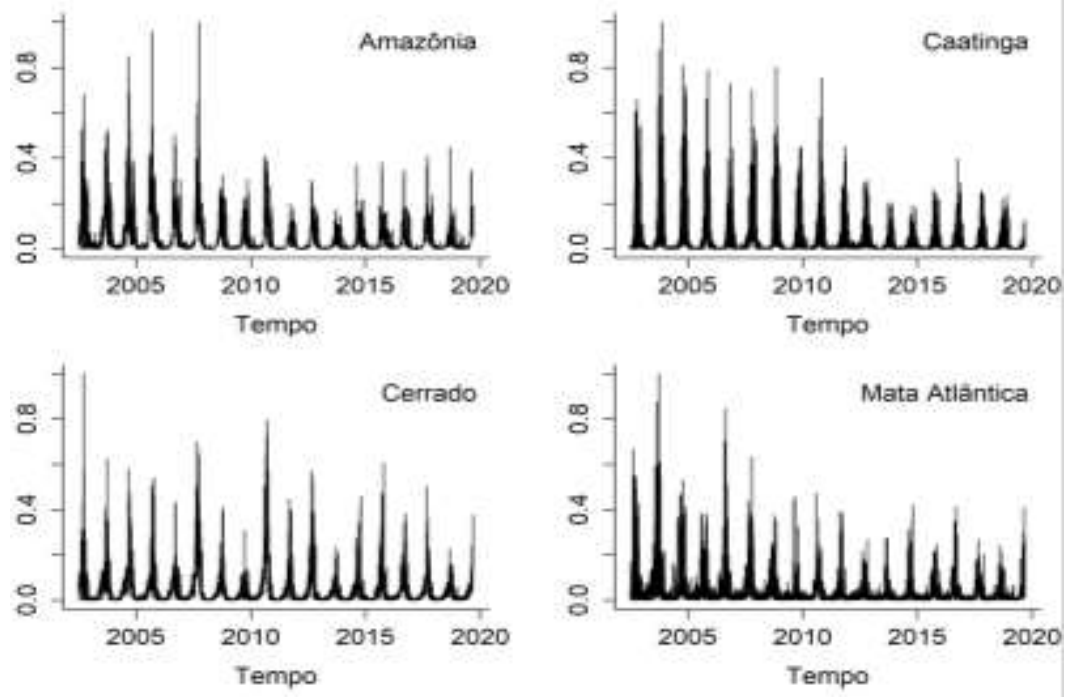

Fonte: Compilação dos autores (2021).

De acordo com os gráficos da Figura 3, identifica-se que, no bioma Amazônia, o mês no qual se obteve a maior ocorrência de focos de calor foi setembro de 2007. No entanto, foi em 2004 que ocorreu o maior registro anual. Tanto o bioma Caatinga quanto o Mata Atlântica tiveram sua maior incidência anual em 2003, enquanto o Cerrado, em 2007. Os maiores quantitativos de focos de calor nesses biomas, entre os dezoito anos analisados, foram observados em 2002, 2003, 2004, 2005, 2007 e 2010, ultrapassando o registro de 300.000 focos, significativamente muito acima da média (241.866 detecções) registrada no País entre 2002 e 2019 (INPE, 2020). O ano de 2010 foi caracterizado por uma seca de grandes proporções na 
floresta amazônica, que foi mais severa do que a seca "uma vez em um século" de 2005 (Marengo, Tomasella, Alves, Soares, \& Rodriguez, 2011). É importante salientar que o bioma Amazônia é o que possui o maior número de focos de calor. Analisando, entretanto, os valores absolutos referentes à quantidade registrada em cada bioma, o Cerrado é o que apresenta a maior concentração por $\mathrm{km}^{2}$, embora a maior incidência de focos ocorra no bioma Amazônia, devido a sua vasta extensão territorial (da Silva, Araújo, Stosic, \& Stosic, 2020).

Como mencionado na seção anterior, a construção dos RPs exige a escolha apropriada dos seguintes parâmetros de recorrência: tempo de retardo $\tau$, obtido por meio de informações mútuas; dimensão de imersão $m$, obtida pelo método dos falsos vizinhos mais próximos (Cao, 1997); e limiar de recorrência $\varepsilon$ para produzir entre $1 \%$ e $5 \%$ do valor de $R R$ como sugerido por Webber e Zbilut (2005). Os valores desses parâmetros obtidos para as séries temporais aqui analisadas encontram-se listados na Tabela 1.

Tabela 1. Valores dos parâmetros de recorrência obtidos para as séries de focos do calor de cada bioma.

\begin{tabular}{lccc}
\hline Bioma & Tempo de atraso $-\tau$ & Dimensão de imersão $-~$ & Limiar $-\varepsilon$ \\
\hline Amazônia & 3 & 10 & 0,008 \\
\hline Caatinga & 3 & 10 & 0,006 \\
\hline Cerrado & 3 & 10 & 0,010 \\
\hline Mata Atlântica & 3 & 11 & 0,017 \\
\hline
\end{tabular}

Fonte: Autores (2021).

Observa-se da Tabela 1 que a dimensão de imersão foi maior para o bioma Mata Atlântica. Esse deve ser, portanto, o valor usado para gerar todos os RPs. No que se refere aos valores do limiar $\varepsilon$, segundo Bastos e Caiado (2011), deve-se utilizar o valor médio para aplicar a Análise de Quantificação de Recorrência. Assim, os RPs foram gerados considerando $m=11$ e $\varepsilon=0,010$.

Os gráficos de recorrência do número de focos de calor nos biomas estudados são apresentados na Figura 4. A tipologia do RP fornece uma visão global do comportamento do sistema, pois permite analisar os estados no espaço de fase em toda série temporal. Para os RPs apresentados na Figura 4, a análise indica que a dinâmica dos focos de calor não é característica de processos aleatórios, tendo em vista que as parcelas de recorrência não exibem apenas pontos únicos isolados.

Observando o gráfico de recorrência do bioma Cerrado, Figura 4(c), nota-se que, no período que começa em aproximadamente meados de 2002 até o final de 2003 ( 1 a 500 dias) e no período de 2005 a 2006 ( 1000 a 1500 dias), há presença de faixas brancas indicando mudanças abruptas na dinâmica do sistema. No RP do bioma Mata Atlântica, Figura 4(d), também se observa uma faixa branca que se destaca em meio às demais no período de 2011 a 2013 ( 3200 a 3900 dias), o que corresponde às oscilações do número de focos de calor na série temporal normalizada desse bioma na Figura 3. Após esse período, a densidade de pontos de recorrência aumenta, mostrando maior similaridade na dinâmica das séries. No RP de focos de calor do bioma Amazônia, Figura 4(a), e Cerrado, Figura 4(c), observa-se tipologia de característica Deriva, em que aparece a diminuição de pontos de recorrência em direção ao canto superior esquerdo e inferior direito. Esse padrão de larga escala indica que o sistema possui parâmetros que variam lentamente (Marwan et al., 2007). O RP de focos de calor do bioma Caatinga, Figura 4(b), apresenta estrutura com aspecto de tabuleiro de xadrez, indicando um processo semiperiódico (Marwan et al., 2007). Os demais RPs também apresentam essa estrutura, traduzindo a periodicidade das séries temporais normalizadas observada na Figura 3. 
Figura 2. Gráficos de Recorrência das séries de focos do calor dos biomas: Amazônia(a), Caatinga(b), Cerrado(c) e Mata Atlântica (d).

(a)

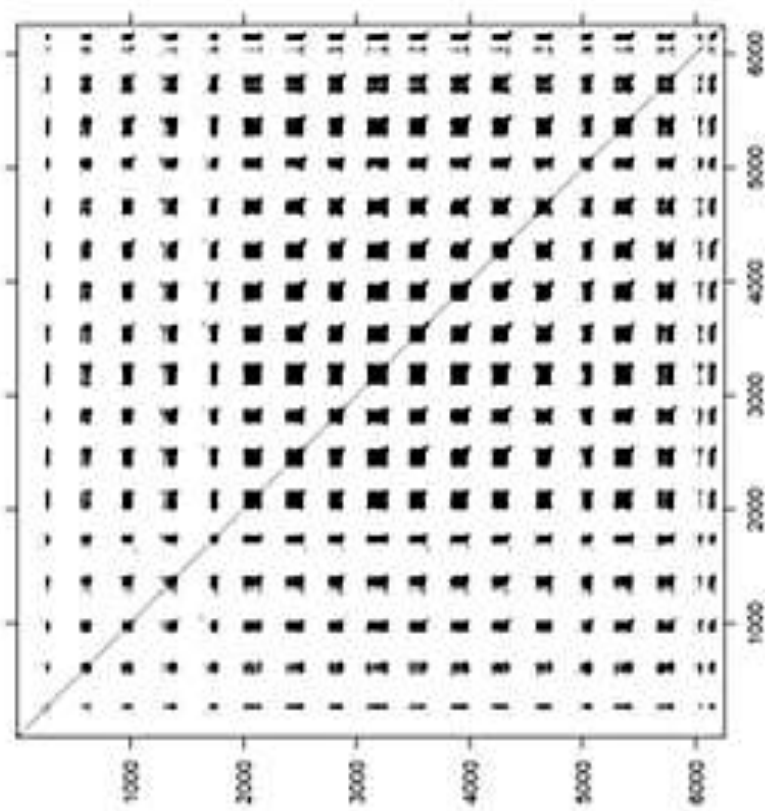

(c)

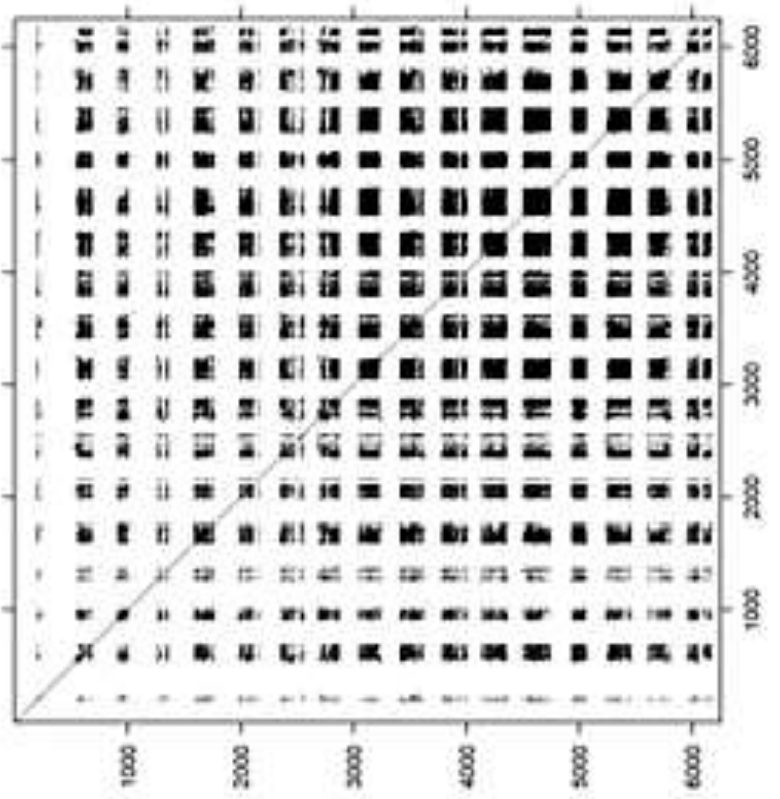

(b)

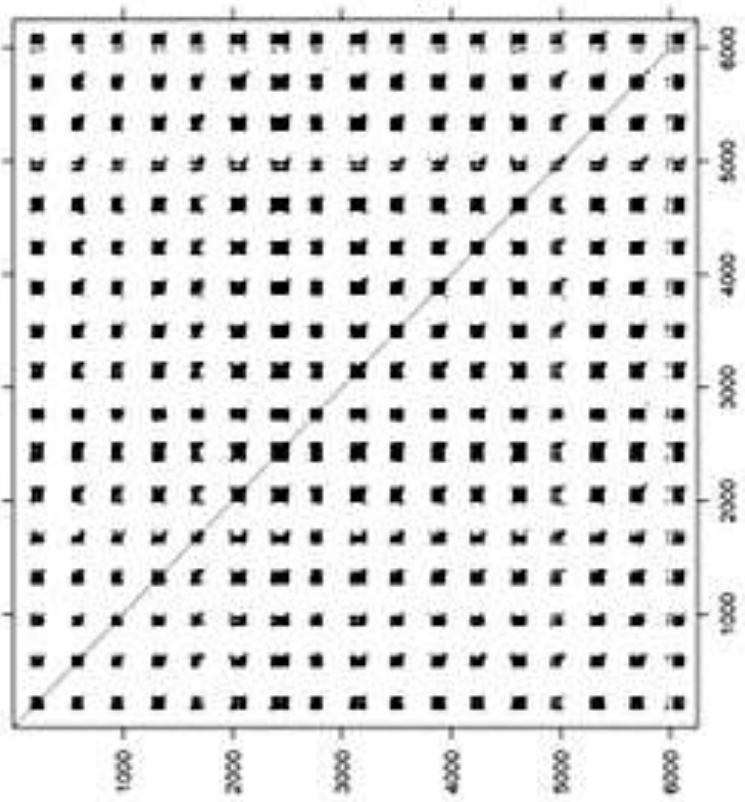

(d)

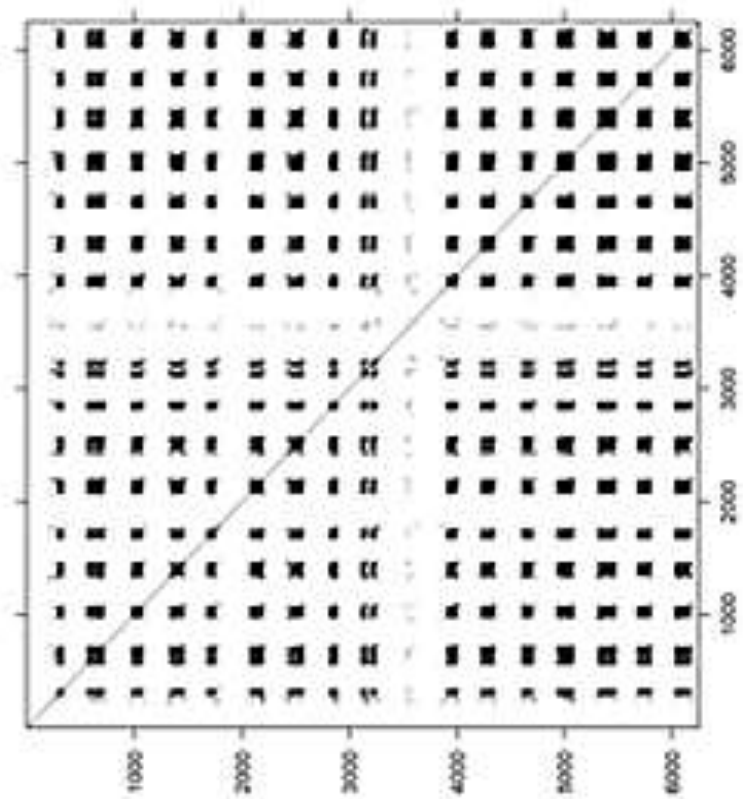

Fonte: Autores (2021).

As medidas de quantificação da Análise de Quantificação de Recorrência dos biomas foram calculadas e seus valores encontram-se listados na Tabela 2. 
Tabela 2. Medidas de quantificação de recorrência obtidas para as séries de focos do calor dos biomas estudados.

\begin{tabular}{|c|c|c|c|c|}
\hline Medida de RQA $\quad$ Bioma & Amazônia & Caatinga & Cerrado & Mata Atlântica \\
\hline Taxa de recorrência $(R E C)$ & 0,05 & 0,077 & 0,03 & 0,001 \\
\hline Determinismo $(D E T)$ & 0,72 & 0,78 & 0,57 & 0,18 \\
\hline Comprimento médio da linha diagonal $(L)$ & 4,13 & 5,31 & 3,79 & 2,18 \\
\hline Maior comprimento da linha diagonal $\left(L_{\max }\right)$ & 161 & 148 & 112 & 36 \\
\hline Laminaridade (LAM) & 0,77 & 0,81 & 0,64 & 0,17 \\
\hline Tempo de permanência $(T T)$ & 5,90 & 8,50 & 5,23 & 2,57 \\
\hline
\end{tabular}

Fonte: Autores (2021).

De acordo com os resultados apresentados na Tabela 2, constatam-se diversas propriedades específicas da dinâmica dos focos de calor nos quatro biomas estudados. Nota-se que os valores de todos os índices são os menores para o bioma Mata Atlântica e os maiores para Caatinga, exceto o valor do comprimento máximo da linha diagonal, $L_{\max }$. Isso indica que as séries do bioma Mata Atlântica são as menos previsíveis (menor determinismo, DET, e comprimento médio da linha diagonal, L), com trajetórias que divergem mais rápido no espaço da fase (menor comprimento máximo da linha diagonal, $L_{\max }$ ), enquanto a série temporal do bioma Caatinga apresenta dinâmica com propriedades opostas (Marwan et al., 2007).

A variável tempo de permanência $(T T)$ tem sido a menos abordada na literatura e, portanto, algumas considerações devem ser feitas. Essa variável, juntamente com a laminaridade $(L A M)$, reflete a persistência de um estado em um determinado intervalo de tempo. Mais especificamente, a variável TT indica o comprimento médio das linhas verticais no gráfico de recorrência. Valores baixos desse comprimento indicam alta complexidade na dinâmica do sistema ("um sistema sem estados laminares”), pois, nessa situação, o sistema permanece por um curto período num estado similar ao do momento anterior (dos Santos, Barroso, de Godoy, Macau, \& Freitas, 2014). Na Tabela 2, constata-se que o tempo de permanência foi relativamente baixo (em relação ao tempo de extensão de RP), indicando o curto tempo em que os sistemas permaneceram num estado laminar. No entanto, esse valor é maior para os dados de focos de calor registrados no bioma Caatinga, indicando que a dinâmica dos focos nesse bioma é mais estável e, mais uma vez, o bioma Mata Atlântica apresentou o menor resultado, indicando menor previsibilidade na dinâmica de focos. É importante ressaltar que os biomas se diferenciam por diversos fatores, como biodiversidade e sensibilidade em relação à ação das queimadas, que pode ser benéfica ou degradante para o ecossistema atingido (Costa \& Rodrigues, 2015).

Os resultados aqui apresentados, obtidos da análise RQA, estão qualitativamente de acordo com os recentemente obtidos por da Silva et al. (2020), que analisaram séries temporais de ocorrência de queimadas detectadas em biomas brasileiros usando o método Horizontal Visibility Graph. O método transforma séries temporais em redes complexas, das quais são extraídos índices topológicos que traduzem a natureza dos processos estocásticos subjacentes. Os resultados obtidos pelos autores mostraram que a dinâmica das queimadas apresentou características de processos estocásticos correlacionados para os biomas Amazônia, Cerrado e Mata Atlântica, entre os quais, as séries de focos de calor do bioma Mata Atlântica apresentaram menor correlação.

\section{Considerações Finais}

O estudo aqui realizado permitiu a análise de aspectos qualitativos e quantitativos da dinâmica de focos de calor dos biomas Amazônia, Caatinga, Cerrado e Mata Atlântica, por meio do método Gráfico de Recorrência (RP) e da Análise de Quantificação de Recorrência (RQA). Essas técnicas possibilitaram a descrição da natureza dos processos estocásticos 
subjacentes e se mostraram satisfatórias quanto à identificação das transições ocorridas nas séries temporais estudadas para os conjuntos de ecossistemas considerados.

Os gráficos de recorrência das séries normalizadas de focos de calor analisadas evidenciaram algumas características observadas na tipologia dos RPs, como: a) os sistemas dinâmicos que originam as séries de focos de calor dos biomas Amazônia e Cerrado possuem parâmetros que variam lentamente e são não-estacionários; b) O bioma Caatinga apresentou RP de estrutura semiperiódica, enquanto o bioma Mata Atlântica resultou num RP com tipologia descontínua, indicando mudanças repentinas na dinâmica do sistema. Quanto à RQA, notou-se que a série temporal do bioma Mata Atlântica mostrou a menor previsibilidade e registrou o maior grau de desordem. O RP do bioma Caatinga, por sua vez, resultou no maior valor para o determinismo e para o comprimento médio da linha diagonal, indicando que a série que o gerou é a mais previsível e apresenta dinâmica de queimadas mais estável.

Em trabalho futuro, planeja-se utilizar o método Gráfico de Recorrência Cruzada (Cross Recurrence Plot) e a Análise de Quantificação de Recorrência correspondente para analisar as correlações entre as séries temporais de queimadas e variáveis climáticas como temperatura, umidade, precipitação e velocidade do vento.

\section{Agradecimentos}

Os autores agradecem à CAPES (Coordenação de Aperfeiçoamento de Pessoal de Nível Superior), ao CNPq (Conselho Nacional de Desenvolvimento Científico e Tecnológico), à FACEPE (Fundação de Amparo à Ciência e Tecnologia do Estado de Pernambuco) e ao INPE (Instituto Nacional de Pesquisa Espaciais) pelo apoio ao desenvolvimento desta pesquisa.

\section{Referências}

Addo, P. M., Billio, M., \& Guegan, D. (2013). Nonlinear dynamics and recurrence plots for detecting financial crisis. The North American Journal of Economics and Finance, 26, 416-435.

Afonso, L. C., Rosa, G. H., Pereira, C. R., Weber, S. A., Hook, C., Albuquerque, V. H. C., \& Papa, J. P. (2019). A recurrence plot-based approach for Parkinson's disease identification. Future Generation Computer Systems, 94, 282-292.

Arbex, M. A., Cançado, J. E. D., Pereira, L. A. A., Braga, A. L. F., \& Saldiva, P. H. D. N. (2004). Queima de biomassa e efeitos sobre a saúde. Jornal Brasileiro de Pneumologia, 30(2), 158-175.

Archibald, S., Roy, D. P., van Wilgen, B. W., \& Scholes, R. J. (2009). What limits fire? An examination of drivers of burnt area in Southern Africa. Global Change Biology, 15(3), 613-630.

Bastos, J. A. \& Caiado, J. (2011) Recurrence quantification analysis of global stock markets. Physica A: Statistical Mechanics and its Applications, v. 390 (7), $1315-1325$.

Bowman, D. M., Balch, J. K., Artaxo, P., Bond, W. J., Carlson, J. M., Cochrane, M. A., \& Pyne, S. J. (2009). Fire in the Earth system. Science, 324(5926), 481-484.

Cao, L. (1997). Practical method for determining the minimum embedding dimension of a scalar time series. Physica D: Nonlinear Phenomena, 110(1-2), 4350 .

Costa, Y. T., \& Rodrigues, S. C. (2015). Efeito do fogo sobre vegetação e solo a partir de estudo experimental em ambiente de cerrado. Revista do Departamento de Geografia, 30, 149-165.

da Silva, J. M., da Silva Araújo, L., Stosic, T., \& Stosic, B. (2020). Análise de séries temporais de focos de calor em biomas brasileiros utilizando o Grafo de Visibilidade Horizontal. Research, Society and Development, 9(9), e308996276-e308996276.

de Santana, L. I. T., da Silva, J. M., da Silva Araújo, L., Moreira, G. R., \& Stosic, T. (2020). Análise de quantificação de recorrência de preços brasileiros do milho, da soja e da carne de frango. Research, Society and Development, 9(10), e9979109461-e9979109461.

Donner, R. V., Balasis, G., Stolbova, V., Georgiou, M., Wiedermann, M., \& Kurths, J. (2019). Recurrence-Based Quantification of Dynamical Complexity in the Earth's Magnetosphere at Geospace Storm Timescales. Journal of Geophysical Research: Space Physics, 124(1), 90-108. 
Research, Society and Development, v. 10, n. 4, e16010413925, 2021

(CC BY 4.0) | ISSN 2525-3409 | DOI: http://dx.doi.org/10.33448/rsd-v10i4.13925

Eckmann, J. P., Kamphorst, S. O., \& Ruelle, D. (1995). Recurrence plots of dynamical systems. World Scientific Series on Nonlinear Science Series A, 16, 441-446.

Facchini, A., Mocenni, C., Marwan, N., Vicino, A., \& Tiezzi, E. (2007). Nonlinear time series analysis of dissolved oxygen in the Orbetello Lagoon (Italy). Ecological modelling, 203(3-4), 339-348.

Faustine, A., Pereira, L., \& Klemenjak, C. (2020). Adaptive weighted recurrence graphs for appliance recognition in non-intrusive load monitoring. IEEE Transactions on Smart Grid, 12(1), 398-406.

Gontijo, G. A. B., Pereira, A. A., Oliveira, E. D. S., \& Júnior, F. W. A. (2011). Detecção de queimadas e validação de focos de calor utilizando produtos de Sensoriamento Remoto. Simpósio Brasileiro de Sensoriamento Remoto, 15, 7966-7973.

Goswami, B., Marwan, N., Feulner, G., \& Kurths, J. (2013). How do global temperature drivers influence each other? The European Physical Journal Special Topics, 222(3), 861-873.

INPE - Instituto Nacional de Pesquisas Espaciais. (2021). Portal do Monitoramento de Queimadas e Incêndios. http://queimadas.dgi.inpe.br/queimadas/portal/informacoes/perguntas-frequentes

Marengo, J. A., Tomasella, J., Alves, L. M., Soares, W. R., \& Rodriguez, D. A. (2011). The drought of 2010 in the context of historical droughts in the Amazon region. Geophysical Research Letters, 38(12).

Marwan, N., Thiel, M., \& Nowaczyk, N. R. (2002). Cross recurrence plot based synchronization of time series. Nonlinear processes in Geophysics, 9(3/4), 325-331.

Marwan, N. (2003). Encounters with neighbours: current developments of concepts based on recurrence plots and their applications. Norbert Marwan.

Marwan, N. (2008). A historical review of recurrence plots. The European Physical Journal Special Topics, 164(1), 3-12.

Marwan, N., Romano, M. C., Thiel, M., \& Kurths, J. (2007). Recurrence plots for the analysis of complex systems. Physics reports, 438(5-6), 237-329.

NSF. Complex Environmental Systems. (2003). http://www.nsf.gov/geo/ere/ereweb/ac-ere/acere_synthesis_rpt_summary.pdf

Rustici, M., Caravati, C., Petretto, E., Branca, M., \& Marchettini, N. (1999). Transition Scenarios during the Evolution of the Belousov-Zhabotinsky Reaction in an Unstirred Batch Reactor. The Journal of Physical Chemistry A, 103(33), 6564-6570.

Soares, R. V., \& Batista, A. C. (2007). Incêndios florestais: controle, efeitos e uso do fogo. Curitiba: Universidade Federal do Paraná.

Zbilut, J. P., \& Webber Jr, C. L. (1992). Embeddings and delays as derived from quantification of recurrence plots. Physics letters A, 171(3-4), 199-203.

Zbilut, J. P., Giuliani, A., \& Webber Jr, C. L. (1998). Detecting deterministic signals in exceptionally noisy environments using cross-recurrence quantification. Physics Letters A, 246(1-2), 122-128. 